\title{
Smoking Today and Stopping Tomorrow: A Limited Foresight Perspective
}

\author{
PHILIPPE JEHIEL \\ ANDREW LILICO
}

CESIFO WORKING PAPER NO. 2603

CATEGORY 11: INDUSTRIAL ORGANISATION

APRIL 2009

\footnotetext{
An electronic version of the paper may be downloaded

- from the SSRN website: www.SSRN.com

- from the RePEc website:

- from the CESifo website:

www.RePEc.org

www.CESifo-group.org/wp
} 


\title{
Smoking Today and Stopping Tomorrow: A Limited Foresight Perspective
}

\author{
Abstract \\ This paper considers an intertemporal decision problem in which the agent has limited \\ foresight. It offers an interpretation of why people may smoke when they are young - and \\ arguably have a short horizon of foresight - and refrain from smoking when they get older - \\ and their foresight is better. \\ JEL Code: D03, D84. \\ Philippe Jehiel \\ Paris School of Economics \\ 48 Bd Jourdan \\ 75014 Paris \\ France \\ jehiel@enpc.fr \\ Andrew Lilico \\ Europe Economics \\ andrew.lilico@europe-economics.com
}

March 23, 2009 


\section{Introduction}

Two important strands of literature have considered the problem of attitude toward smoking. On the one hand, the theory of addiction suggests that the more you have smoked in the past, the more smoking is preferred to nonsmoking (even though in absolute terms it may be worse than having never smoked). According to this theory, if agents are fully rational, they should anticipate this and refrain from smoking in the first place (if the long run consequences of smoking today are sufficiently bad). On the other hand, the theory of hyperbolic discounting postulates that agents keep valuing immediate rewards excessively high as compared with what standard exponential discounting would deliver. According to this theory, even if agents are fully rational they may prefer smoking today because this delivers an instantaneous pleasure that may offset the long run consequences (according to the current period criterion).

Neither of these approaches challenges the cognitive ability of agents to understand the consequences of their current consumption decisions for their future consumption decisions. We propose to revisit the problem of the attitude toward smoking by considering agents that have limited foresight and understand only how their current consumption decisions affect the pattern of consumption over a limited future (as opposed to the entire future). In our model, discounting takes the usual exponential form and we allow for changing preferences that are affected by past consumptions (as in models of addiction).

Our main interest lies in showing the effects of a change in the horizon of foresight of agents. As it turns out, agents with short or intermediate horizons of foresight might smoke or cycle between smoking and non-smoking whereas agents with sufficiently long horizons of foresight will refrain from smoking, as we assume that non-smoking is the optimal plan of action under perfect foresight. The limited foresight perspective adopted in this paper has policy implications that differ from those suggested by other approaches - in particular the hyperbolic discounting approach. In our framework, a very long horizon of foresight is always good for the agent. So, if it were possible to make agents more aware of the long run effects of their current consumption decisions, this would unambiguously lead to a welfare improvement in our setup. This is not so in the hyperbolic discounting paradigm in which it is assumed that agents have perfect foresight (there taxes on cigarettes may be the only tool to align the instantaneous criterion of the agent with his long 
run well being as assumed by the planner). ${ }^{1}$

Studies of attitudes towards smoking are an important source of evidence that agents are subject to limited foresight, with their foresight horizons differing depending on how experienced they are. For example, if agents were rational and there were no material shocks affecting the average tendency to smoke, then people's expectations of their likelihood of being smokers in future years should, on average, correspond to the numbers of these individuals that actually are smokers. Yet, according to US DHHS 1994 data, as reported in Loewenstein et al. (2003), only 15 percent of high school students that were occasional smokers (whom we interpret as less experienced people) predicted that they might be smoking in five years, when in fact 42 percent were still smoking five years later. By comparison, 68 percent of high school students that were heavy smokers (whom we interpret as more experienced people) predicted that they would still smoking be smoking in five years, while 80 percent were still smoking five years later. Assuming that there were no material shocks increasing the aggregate tendency to smoke in the period of this study, these results constitute evidence for limited foresight among young smokers, with the foresight error being far less severe for heavy (more experienced) young smokers than for light (less experienced) young smokers.

Studies of smoking also provide indicative evidence regarding the effect of age on forecasting abilities. For example, we note the following from the UK Department of Health Smoking-related behaviour and attitudes survey, 2003 and the National Statistics database of trends in smoking, 1974-2003. First we note that significantly higher proportions of the population smoke when 20-24 than when older - for example, in 2003, 36 per cent compared with 25 per cent of 50-59 year-olds and and only 15 per cent of 60 and overs. Next, we note that significant numbers of people give up when relatively young. Some 20-25 per cent of smokers give up between the ages of 20-24 and 35-49 (e.g. for 200336 per cent of 20-24 year-olds smoke, whilst only 30 per cent of 35-49 year-olds smoke). Some of this giving up occurs between 24 and 35 (In the data, after 1982, in every year except 2000, there are fewer smokers at 25-34 than at 20-24). Though many other factors change as one ages (e.g. children may arrive), the important scale of giving-up occurring between the very young and slightly older age-groups is at least consistent with the view

\footnotetext{
${ }^{1}$ In our setup, taxes on cigarettes could hurt agents with perfect foresight who would find it optimal to smoke taking into account all the consequences of smoking.
} 
that greater experience, of some sort, is important. Next we note that in Table 3.21 of Smoking-related behaviour and attitudes, 2003, 57 per cent of 16-24 year old smokers who intend to stop smoking are reported to respond they might or might not be smoking in a year, whereas the same figure is only 35 per cent for 25-44 years old - perhaps indicative that a high proportion of this young age group has a horizon of foresight of less than a year whilst more of the older age group has a longer horizon. Of course, more systematic econometric investigations would be required to produce robust results, but, taken together, our theory linking forecasting abilities to smoking attitudes seems, at least on the face of it, consistent with these data.

In the rest of the paper, we define a solution concept to describe the consumption choices of agents who are subject to limited foresight. Our modelling approach follows Jehiel (1995): Agents are assumed to make correct forecasts within their of horizon of foresight, ${ }^{2}$ and they extrapolate from their discounted sum of payoffs obtained within their horizon of foresight to form a simple decision making criterion based on the limited forecast. We also suggest a refinement of the solution concept based on the idea that agents tend to prefer controlling their plans of action as far ahead as possible. Next we use our setup to generate examples in which the decision of whether to smoke or not to smoke is sensitive to the horizon of foresight and/or in which cycling behaviour between smoking and non-smoking may emerge as an equilibrium phenomenon. Then we relate our approach to other approaches in the literature and offer a conclusion.

\section{Setup}

A decision-maker faces infinitely-many stages, with a decision to make at each stage. His decision in each period $t$ consists in a choice of action $a_{t} \in A$ where $A$ is a finite choice set. His preferences are captured by

$$
\sum_{t=0}^{\infty} \delta^{t} U\left(a_{t}, \omega_{t}\right)
$$

where $\omega_{t} \in \Omega$ is a state variable, $U\left(a_{t}, \omega_{t}\right)$ is the flow of payoff derived by the agent in period $t$, and $\delta$ is the discount factor between periods.

\footnotetext{
${ }^{2} \mathrm{~A}$ rationale for the correctness assumption might be based on learning considerations, see Jehiel (1998).
} 
The state evolves according to a deterministic process mapping the period $t$ profile of state $\omega_{t}$ and action $a_{t}$ onto the period $t+1$ state $\omega_{t}$ (i.e., $\left.\left(\omega_{t}, a_{t}\right) \rightarrow \omega_{t+1}\right) .^{3}$ Thus, the state at period $t+k$ can be written as a function $\omega_{t+k}\left(\omega, a^{0}, a^{1}, \ldots, a^{k-1}\right)$ where $\omega_{t}=\omega$ denotes the period $t$ state and $a_{t+l}=a^{l}$ the period $t+l$ action.

We note that in a standard paradigm with perfect foresight there is no "time inconsistency" problem - that is to say, once a plan of what to do in the future is formed, when the decision-maker gets there he doesn't change his mind. What he thought would be the best thing to do in the future continues to be the best thing to do once the future becomes the present. This is due to the fact that discounting takes an exponential form so that the marginal rate of substitution (in terms of overall preference) between an increase of utility in period $t$ and an increase of utility in period $t+k$ does not change with the time period $t$. This is a generalization of a result first noted by Strotz (1956).

\section{The limited foresight approach}

We turn now to describing the decision making of an agent with limited foresight. We first review the basic concepts and then offer some preliminary results.

\subsection{Concepts}

In a limited foresight problem, the decision-maker can see ahead only $n$ periods (including the current period), and forms no view as to what happens beyond the horizon of foresight. The following definition (in the spirit of Jehiel 1995) describes how an agent with limited foresight behaves.

Definition 1 A stream of actions $\left\{a_{t}(\omega)\right\}_{t, \omega}$ is a "limited foresight $n$-equilibrium"

\footnotetext{
${ }^{3}$ Our insights would extend to more general stochastic processes. We chose a deterministic formulation for expositional purposes.
} 
(or "LFE-n") if and only if $, \forall t, \omega$,

$$
a_{t}(\omega)=\arg \max _{a}\left[U(a, \omega)+\sum_{k=1}^{n-1} \delta^{k} U\left(\widehat{a}^{k}, \omega^{k}\right)\right]
$$

where $\forall k \leq n-1,{ }^{5}$

$$
\begin{aligned}
\widehat{a}^{k} & =a_{t+k}\left(\omega^{k}\right) \\
\omega^{k} & =\omega_{t+k}\left(\omega, a, \widehat{a}^{1}, \ldots, \widehat{a}^{k-1}\right)
\end{aligned}
$$

The idea behind the limited foresight equilibrium is that at each point in time and whatever the state $\omega$ the agent understands the effect of his current consumption decision over the next $n$ periods but not further ahead. So at time $t$, his foresight horizon covers consumption decisions from periods $t+1$ to $t+n-1$, and as one moves on to the next period $t+1$, his foresight horizon covers consumption decisions from periods $t+2$ to $t+n$. Such an idea of rolling (limited) foresight is reminiscent of how a chess player would usually simplify the analysis of the huge game tree generated after most non-trivial board positions, and we believe it is likely to apply to most interactions with sufficiently complex decision/game trees.

We assume that the agent's forecast is correct within his horizon of foresight, which is reflected in the fact that the period $t$ expectation $\widehat{a}^{k}$ for period $t+k$ (rightly) corresponds to the effective agent's consumption decision in period $t+k$ given the consumption decision $a$ considered in period $t$ and the true (chosen) pattern of consumptions from then on, i.e $\widehat{a}^{k}=a_{t+k}\left(\omega^{k}\right)$. The correctness assumption is clearly an extreme and idealized assumption, and would be problematic if we thought of it as a result of introspective calculus. $^{6}$ Our view of this assumption is in terms of learning. The agent

\footnotetext{
${ }^{4}$ We restrict attention to deterministic action schemes that may only depend on the time period $t$ and the current state $\omega$, hence $a_{t}(\omega)$. Following Jehiel (1995), it can be shown that this is without loss of generality, as long as the agent's choice of action is deterministic and cannot (directly) depend on actions that took place more than $N$ periods earlier (i.e., as long as the agent has bounded recall).

${ }^{5}$ Note that $\omega^{k}$ depends on $a$ for $k \geq 1$, so $\widehat{a}^{k}$ depends on $a$ too.

${ }^{6}$ If the agent were able in period $t+1$ to make correct forecast till period $t+n$ as a result of introspective calculus it seems hard to justify why he could not have made this calculus in period $t$ thus implying that his period $t$ 's forecast should cover one more period than we assume. By a simple unravelling argument, this line of thought should imply that the agent has perfect foresight over the entire future.
} 
is viewed as inferring from his own past experiences and the experiences of his fellow smokers and non-smokers his smoking attitude in a limited time frame. For example, if our agent has a one-year horizon of foresight, he is viewed as correctly assessing his ability to stop smoking within a year time when he starts or continues smoking now, and he would also correctly assess his chance of smoking again within a year time if he refrains from smoking for a week time. But, the agent would have no correct expectation about his smoking attitude beyond one year. The agent is then viewed as making his current period consumption decision so as to maximize his expected payoff within his foresight horizon. That is, the agent somehow ignores the time periods over which he has no foresight. ${ }^{7}$ It seems reasonable to suppose that an older or more experienced agent is likely to have a longer horizon of foresight (as in chess), and in our application we will be interested in the effect of increasing the horizon of foresight on the consumption patterns that can possibly emerge in equilibrium (i.e. that can be stable points of the implicit learning model suggested above).

In the above equilibrium concept, the agent is assumed to choose his current consumption only (as opposed to a consumption plan within his horizon of foresight). The limited horizon forecast is only a means through which the agent can elaborate the best decision for the current period; it is not a choice of the agent. ${ }^{8}$ The next definition (which is new to this paper) suggests considering a scenario in which the agent would choose his consumption decisions within the next $p$ periods with the additional constraints that the agent does not change his mind when these periods come about (i.e. so as to avoid time inconsistencies issues).

Definition 2 A stream of actions $\left\{a_{t}(\omega)\right\}_{t, \omega}$ is a "p-controlled n-equilibrium" (or "LFE- $(p, n)$ ") if and only if, $\forall t, \omega$

A. $\left(a_{t}, \ldots, a_{t+p-1}\right)=\arg \max _{a^{0}, \ldots, a^{p-1}}\left[\sum_{k=0}^{p-1} \delta^{k} U\left(a^{k}, \omega^{k}\right)+\sum_{k=p}^{n-1} \delta^{k} U\left(\widehat{a}^{k}, \omega^{k}\right)\right]$

B. $\widehat{a}^{k}=a_{t+k}\left(\omega^{k}\right)$ for $p-1<k \leq n-1$ and

\footnotetext{
${ }^{7}$ There are alternative views. For example, an agent could be parameterized by how optimistic he is about what is to come beyond his horizon of foresight. This would give rise to alternative criteria (see Jehiel (2001) for the study of such alternative criteria in the context of repeated games).

${ }^{8}$ This modeling device is in the spirit of Strotz (1956), and it is meant to avoid time inconsistency problems.
} 
C. $a^{k}=a_{t+k}\left(\omega^{k}\right)$ for $k \leq p-1$ where for all $k$,

$$
\begin{aligned}
\omega^{k} & =\omega_{t+k}\left(\omega, b^{0}, \ldots, b^{k-1}\right) \text { and } \\
b^{k} & =\mid \begin{array}{l}
a^{k} \text { for } k \leq p \\
\widehat{a}^{k} \text { for } k>p
\end{array}
\end{aligned}
$$

In this case we divide the foresight horizon into a period over which the decision-maker plans what to do, $p$ (which we shall refer to as his "planning horizon"), and the period over which he merely has exogenous expectations of what he will do, $n-p$.

The interpretation of Definition 2 is as follows. Condition A means that in every period $t$ and in all states $\omega$, the agent chooses an optimal plan $a^{0}, \ldots, a^{p-1}$ over his planning horizon given his expectations $\widehat{a}^{p}, \ldots, \widehat{a}^{n-1}$ about what will happen next within his horizon of foresight. Condition B expresses the idea that expectations $\widehat{a}^{p}, \ldots, \widehat{a}^{n-1}$ are correct, while condition $\mathrm{C}$ expresses the idea that within his planning horizon the agent does not change his mind. That is, when the agent reaches period $t+k$, he finds it optimal to do what he had planned to do at this period $k$ periods earlier, i.e. at period $t^{9}$.

When the agent has perfect foresight $(n=+\infty)$, it is readily verified that an $\operatorname{LFE}-(p,+\infty)$ exists and that it coincides with the standard perfect foresight optimal plan. This is a simple adaptation/generalization of Strotz (1956)' s result (it is due to the exponential character of the discounting). It is also immediate to see that a limited foresight equilibrium LFE- $n$ corresponds to a $p$-controlled $n$-equilibrium or LFE- $(p, n)$ with a planning horizon of $p=1$ and that any $\operatorname{LFE}-(p, n)$ is also a LFE- $n$.

In general, while an LFE- $(1, n)$ always exists, an $\operatorname{LFE}-(p, n)$ need not exist when $p>1 .{ }^{10}$ The next definition suggests looking at those LFE- $(p, n)$ which have a maximal planning horizon $p$.

\footnotetext{
${ }^{9}$ Rubinstein (1998) suggests as an alternative to Jehiel (1995)'s approach a concept in which the planning horizon $p$ coincides with the horizon of foresight $n$ and condition $\mathrm{C}$ is dropped. But, this in general would result in the decision maker adopting decisions based on plans that are not followed afterwards, which sounds undesirable (see also Jehiel (1998) and Lilico (2002) for further discussion of this point).

${ }^{10}$ For example, consider a case in which a decision-maker must choose between going up and down at an initial node, and can see that if he goes down the problem ends, but if he goes up he will later have the option of going up or down at the next stage, but can see no further ahead than that. There are stage payoffs of 1 for going Down at the first node and 0 for going Up, and 2 and 0 for going Up or Down (respectively) at the second node (reached only if Up is chosen at the first node). Suppose that if he goes up he will
} 
Definition 3 A stream of actions $\left\{a_{t}(\omega)\right\}_{t, \omega}$ is a "best-controlled n-equilibrium" (or "LFEB-n") if and only if it is an LFE- $(p, n)$ and there is no LFE- $\left(p^{\prime}, n\right)$ with $p^{\prime}>p$.

The motivation for a best-controlled $n$-equilibrium is that an agent may find it desirable to feel he has as much control over his planning scheme as possible subject to the constraint that he does not change his mind (relative to plans made earlier $)^{11}$.

\subsection{Preliminary Results}

Proposition 1 There always exists an LFE-n. All LFE-n are cyclical - i.e.

$$
\exists k \text { s.t. } \forall \omega, t, a_{t+k}(\omega)=a_{t}(\omega)
$$

Proof. (Sketch) Start from any profile of $n$-expectations, i.e. $f_{0}(\omega, a) \in$ $A^{n}$. For any $\omega, a_{f_{0}}^{*}(\omega)$ is a best-action given $f$. Construct $f_{-1}$ as follows:

For any $\omega$, if $a$ is chosen, let $\omega^{\prime}$ denote the new state. Take the sequence of actions generated by $a_{f_{0}}^{*}\left(\omega^{\prime}\right)$ and $f_{0}\left(\omega^{\prime}, a_{f_{0}}^{*}\left(\omega^{\prime}\right)\right)$. Take the truncation of these to the first $n$ ones. Define this to be $f_{-1}(\omega, a)$. Define recursively $f_{-k}(\omega, a)$. At some point, because everything is finite, $f_{-k}$ will correspond to $f_{-k^{\prime}}, k \neq k^{\prime}$. This allows us to show existence and the cyclical nature of limited foresight plans. N.B. this is analogous to Jehiel (1995).

A simple corollary of Proposition 1 is of course that an LFEB- $n$ always exists. This is because LFE-1 and LFE- $(1,1)$ are equivalent concepts. So even though an LFE- $(p, n)$ need not exist when $n \geq 2$ and $p \geq 2$, an LFEB- $n$ always exists.

then be able to see to the end of the problem, understanding that going Up will lead to a further choice of payoffs of -3 (for Up) or -5 (for Down), whilst going Down will deliver a choice between payoffs of 29 (for Up) and 0 (for Down). In this situation, the LFE-1 equilibrium strategy is (Down, Down, Up, Up). However, there is no LFE- $(2,2)$ strategy. At the first node, the optimal 2-plan is (Up, Up), but upon reaching the next node, the agent would choose Down and not Up, violating the constraint that he should not change his mind within his planning horizon.

${ }^{11}$ Clearly, a best-controlled $n$-equilibrium is an LFE- $n$ equilibrium and it can thus be viewed as a refinement of it. 


\section{To smoke, or not to smoke? That is the question...}

Now we shall employ the concepts we have introduced to address the question of why some people might smoke when young, then give up when older. In so doing we shall interpret a younger (resp. older) person as one with shorter (resp. longer) horizon of foresight.

We shall make the following simplifying assumptions:

A1) $A=\{D, S\}$ : there are two actions, which we shall interpret as Don't Smoke and Smoke.

A2) $\omega_{t}=\left(a_{t-1} a_{t-2} \ldots a_{t-m}\right)$ : the state variable is defined to be the $m$ previous period actions.

A3) $\exists \bar{\delta}$ such that, for all $\delta \geq \bar{\delta}$, there is one perfect foresight optimal plan, which is $a_{t}(\omega)=D, \forall \omega, t$.

In the sequel we shall analyze how the plan of an agent who has limited foresight varies with his horizon of foresight. A3 tells us that an agent with perfect foresight chooses $D$ in all states $\omega$ and in all periods. In light of the smoker's problem, A3 sounds plausible to us.

More precisely, we wish to address the following questions. 1) Under what conditions on the horizon of foresight and the payoff structure are the stationary plans of always choosing $S$ or always choosing $D$ sustainable? 2) Can the two stationary plans just defined be simultaneously sustainable? 3) Can cycling between $D$ and $S$ be sustainable when neither stationary plan is? 4) Can cycling behavior be sustainable as well as some stationary plan?

In the rest of this Section, we answer these questions through a series of examples. Stationary plans are referred to as

Plan $D: a_{t}(\omega)=D, \forall \omega, t$

Plan $S: a_{t}(\omega)=S, \forall \omega, t$

Non-stationary plans involving cycling behavior will be introduced later. For each plan that we will consider, we are interested in whether or not there exists $\bar{\delta}$ such that for all $\delta \geq \bar{\delta}$ this plan is sustainable (as LFE- $n$ or LFEB- $n$ ). This is equivalent ${ }^{12}$ to checking the sustainability of such a plan (as LFE- $n$ or LFEB- $n$ ) in the special case $\delta=1$. From here on we shall assume that $\delta=1$.

\footnotetext{
${ }^{12}$ up to indifferences (that we assume are not present here) in the limit as $\delta$ goes to 1.
} 


\subsection{When the horizon of foresight is long enough}

We first show that when the horizon of foresight $n$ is strictly greater than the hindsight dependence $m$, the only possible stationary plan is $D$.

Proposition 2 For all $n \geq m+1$, Plan D (but not $S$ ) is an LFE-n.

This result follows from the observation that stationary LFE- $n$ are not affected by the horizon of foresight $n$ as long as $n \geq m+1$ :

Lemma 1 For all $n \geq m+1$, Plan $a$ is an LFE-n if and only if it is an $L F E-(m+1)$.

And only plan $D$ can be an $\operatorname{LFE}-(m+1)$ :

Lemma 2 Plan $D($ but not $S)$ is an LFE- $(m+1)$.

The above proposition clearly rules out the possibility that plan $S$ is sustainable for long enough horizons of foresight. However, it does not rule out the possibility that cycling behavior is also sustainable for $n \geq m+1$. As it turns out, cycling behaviour may be sustainable together with plan $D$ even for arbitrarily large values of the horizon of foresight $n$. This is illustrated through the following example in which $m=1$ and the payoffs (reading states horizontally and this-period actions vertically) are as given in Table 1:

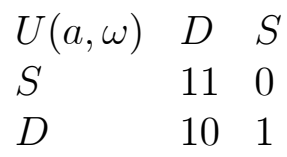

Table 1

Such payoffs capture situations in which the decision maker prefers smoking to not smoking when he has not smoked in the recent past, but when he has smoked in the recent past he dislikes both smoking and non-smoking and he dislikes smoking even more (say he feels disgust). It is readily verified that plan $D$ is the optimal perfect foresight solution because starting to smoke triggers a great payoff loss to the agent. Thus, assumption A3 holds.

Consider the cyclical plan in which the agent smokes whenever he did not smoke in the last period and he refrains from smoking otherwise: 
Cyclical plan: for all $t, a_{t}(\omega=S)=D$ and $a_{t}(\omega=D)=S$.

Clearly, the cyclical plan is an LFE-1, i.e, it is a stable plan when the agent is myopic (this is because $U(D, S)>U(S, S)$ and $U(S, D)>U(D, D)$ ). Interestingly, the cyclical plan is also an LFE- $n$ for all odd values of the horizon of foresight $n$. To see this, let $n=2 k+1$ and observe that the equilibrium condition boils down to the following two inequalities:

1) $U(D, S)+k[U(S, D)+U(D, S)] \geq U(S, S)+k[U(D, S)+U(S, D)]$

2) $U(S, D)+k[U(D, S)+U(S, D)] \geq U(D, D)+k[U(S, D)+U(D, S)]$

These two conditions simplify into the conditions for the cyclical plan to be an LFE-1, which hold as explained above. Observe however that the cyclical plan is not an LFE- $n$ whenever $n$ is an even number. (This is because say $U(S, D)+U(D, S)<U(D, D)+U(S, D)$.)

Thus, even as the agent's horizon of foresight $n$ gets very large it may be that the cyclical plan defined above is sustainable as an LFE- $n$, even though this cyclical plan cannot be an LFE- $n$ for all arbitrarily large values of $n$.

A more systematic investigation as to when cycling behaviour may occur for arbitrarily large horizons of foresight deserves further research. We suspect that for cycling to be sustainable in such a way it must be that in at least one state $\omega, U(S, \omega)<U(D, \omega)$, which some smokers might dispute to be a plausible assumption. (As non-smokers, we tend to find such an assumption quite plausible! $)^{13}$ The remaining examples will have the feature that for all states $\omega, U(S, \omega)>U(D, \omega)$ so that plan $S$ is the only LFE-1.

\subsection{When the horizon of foresight is short}

We now consider situations in which the horizon of foresight $n$ is smaller than $m+1$. We shall focus attention on proving that for such horizons of foresight, there are a number of scenarios in which Plan $S$ would be optimal, and some in which Plan $D$ would not be optimal. We offer the interpretation that those who smoke may face decision-problems like those below.

In the sequel, we shall specialize to the case of $m=2$.

\footnotetext{
${ }^{13}$ As a side comment, casual observation suggests that even some experienced agents give the impression of cycling between smoking and non-smoking, and then the impression of quitting cigarettes completely, before starting again cycling between smoking and not smoking. We propose to interpret such patterns of behaviour through the lens of continuously increasing horizons of foresight with payoff structures such as those shown in Table 1 .
} 
When Plan $S$ (but not $D$ ) is a limited foresight equilibrium: Consider the following payoffs (reading states horizontally and this-period actions vertically):

$\begin{array}{lllll}U(a, \omega) & D D & D S & S D & S S \\ S & 11 & 8 & 11 & 9 \\ D & 10 & 4 & 3 & 0\end{array}$

Table 2

This system of payoffs guarantees that if the agent is patient enough $(\delta$ close to 1), the optimal perfect foresight plan is to never smoke, i.e. Plan $D$. Hence, Assumption A3 is satisfied. The system of payoffs is also such that in all states $\omega, U(S, \omega)>U(D, \omega)$ so that plan $S$ is the only LFE-1.

We next consider a situation in which the agent predicts one period ahead but not more, i.e $n=2$. We observe that Plan $D$ is not an LFE-2. This is because: ${ }^{14}$

$$
U(D, S D)+U(D, D S)<U(S, S D)+U(D, S S) .
$$

Thus, anticipating he will not smoke in the next period, the agent would be better off smoking today if yesterday he smoked and the day before he did not. Thus plan $D$ is not an LFE-2.

Finally, Plan $S$ is an LFE-2. This is because the following system of inequalities is satisfied (checking all possible states): ${ }^{15}$

1) $2 U(S, S S)>U(D, S S)+U(S, D S)$

2) $U(S, D S)+U(S, S D)>U(D, D S)+U(S, D D)$

3) $U(S, D D)+U(S, S D)>U(D, D D)+U(S, D D)$

4) $U(S, S D)+U(S, S S)>U(D, S D)+U(S, D S)$

\footnotetext{
${ }^{14} 3+4=7<11=11+0$

15 1) $2 * 9=18>8=0+8$

2) $8+11=19>15=4+11$

3) $11+11=22>21=10+11$

4) $11+9=20>11=3+8$
} 
Hence, whatever the state, the agent finds it optimal to choose $S$ today if he anticipates he will choose $S$ tomorrow.

In the above example, Plan $D$ is an LFE-3, but Plan $S$ is not (see Proposition 2). Additionally, Plan $S$ is an LFE-2, but Plan $D$ is not (see above calculations). Thus if decision-makers faced such preferences at a time when their foresight horizon was relatively short (2) they would smoke. If subsequently (and unexpectedly) their foresight horizon later became longer they would switch to not smoking. We shall discuss this further below.

Cycling as a limited foresight equilibrium:

Consider the following payoffs

$\begin{array}{lllll}U(a, \omega) & D D & D S & S D & S S \\ S & 95 & 20 & 106 & 13 \\ D & 90 & 15 & 100 & 0\end{array}$

Table 3

It is readily verified that Plan $D$ is the optimal perfect foresight solution so that Assumption A3 holds. Besides, in all states $\omega, U(S, \omega)>U(D, \omega)$ so that plan $S$ is the only LFE-1, and $U(S, \omega)-U(D, \omega)$ is weakly increasing as $\omega$ moves from $D D$ to $D S$ and $S D, S S$, which reflects the idea that smoking relative to non-smoking is all the more enjoyable that one has smoked more in the (recent) past, i.e. there is something like addiction.

Consider now a foresight $n=2$. Plan $D$ is not an LFE-2 because

$$
U(S, D D)+U(D, S D)>2 U(D, D D)
$$

Plan $S$ is not an LFE-2 because

$$
U(D, S D)+U(S, D S)>U(S, S D)+U(S, S S)
$$

We will show that cycling between smoking and not smoking can arise in a limited foresight equilibrium.

Specifically, consider the following plan:

For all $t, a_{t}(S D)=D$ and $a_{t}(D D)=a_{t}(D S)=a_{t}(S S)=S$. 
This plan gives rise to two possible consumption patterns: $D S D S D S$ where there is alternation between smoking and non-smoking and $S S S S$ in which the agent keeps smoking every period. It is readily verified that the above cyclical plan is an LFE-2 as

1) $U(S, D D)+U(D, S D)>U(D, D D)+U(S, D D)$

2) $U(S, D S)+U(D, S D)>U(D, D S)+U(S, D D)$

3) $U(D, S D)+U(S, D S)>U(S, S D)+U(S, S S)$

4) $U(S, S S)+U(S, S S)>U(D, S S)+U(S, D S)$

This illustrates that cycling may emerge for intermediate horizons of foresight when plan $D$ is the perfect foresight solution, and neither plan $S$ nor plan $D$ is a limited foresight equilibrium. ${ }^{16}$

\section{When Plan $S$ is a better-controlled plan than Plan $D$ :}

Another type of scenario in which people might smoke is one in which, although both Plans would be equilibria if we took our future behaviour as exogenous, if we aim to control what we do in the future as far ahead as possible (as in the LFEB- $n$ concept), there are advantages to smoking.

Consider the following payoffs:

$\begin{array}{lllll}U(a, \omega) & D D & D S & S D & S S \\ S & 4 & 5 & 14 & 7 \\ D & 8 & 10 & 6 & -2\end{array}$

Table 4

Again, it can be checked that Plan $D$ is the only optimal perfect foresight plan and plan $S$ is the only LFE-1. Besides, $U(S, \omega)-U(D, \omega)$ is increasing as $\omega$ moves from $D D$ to $D S$ and $S D, S S$, which reflects the idea of addiction.

Also, both Plans $D$ and $S$ are LFE-2.

\footnotetext{
${ }^{16}$ Note however that the above cyclical plan is not an LFE- $n$ for any $n>2$; this is because at state $\omega=S S$ the agent would choose $D$ rather than $S$ (so as to benefit from $U(D, S D))$.
} 
For Plan $D$ this is because ${ }^{17}$

1) $2 U(D, D D) \geq U(S, D D)+U(D, S D)$

2) $U(D, S D)+U(D, D S) \geq U(S, S D)+U(D, S S)$

3) $U(D, S S)+U(D, D S) \geq U(S, S S)+U(D, S S)$

4) $U(D, D S)+U(D, D D) \geq U(S, D S)+U(D, S D)$

For Plan $S$ this is because ${ }^{18}$

5) $2 U(S, S S)>U(D, S S)+U(S, D S)$

6) $U(S, D S)+U(S, S D)>U(D, D S)+U(S, D D)$

7) $U(S, D D)+U(S, S D)>U(D, D D)+U(S, D D)$

8) $U(S, S D)+U(S, S S)>U(D, S D)+U(S, D S)$

Although both Plans $D$ and $S$ are LFE-2s, only Plan $S$ is an LFEB-2. We know that with $p=1$ both Plans are LFE- $(1,2)$ s, since an LFE- $(1,2)$ is formally equivalent to an LFE-2. Thus for Plan $S$ to be an LFEB-2 but Plan $D$ not, Plan $S$ must be an LFE- $(2,2)$ but Plan $D$ not. For Plan $S$ to be an LFE- $(2,2)$ it should be that for all states the best 2-plan is to Smoke in the current and next period. This requires the following inequalities in addition to those required for LFE-2:

9) $2 U(S, S S) \geq U(D, S S)+U(D, D S)$

10) $2 U(S, S S) \geq U(S, S S)+U(D, S S)$

11) $U(S, D S)+U(S, S D) \geq U(D, D S)+U(D, D D)$

12) $U(S, D S)+U(S, S D) \geq U(S, D S)+U(D, S D)$

13) $U(S, D D)+U(S, S D) \geq U(D, D D)+U(D, D D)$

14) $U(S, D D)+U(S, S D) \geq U(S, D D)+U(D, S D)$

15) $U(S, S D)+U(S, S S) \geq U(D, S D)+U(D, D S)$

\footnotetext{
17 1) $2 * 8=16>10=4+6$

2) $6+10=16>12=14-2$

3) $-2+10=8>5=7-2$

4) $10+8=18>11=5+6$

185) $2 * 7=14>3=-2+5$

6) $5+14=19>14=10+4$

7) $4+14=18>12=8+4$

8) $14+7=21>11=6+5$
} 
16) $U(S, S D)+U(S, S S) \geq U(S, S D)+U(D, S S)$

It is easily verified that these all hold.

In contrast, the equivalent inequalities for Plan $D$ do not all hold. In particular, we have ${ }^{19}$ :

$$
U(D, S D)+U(D, D S)<U(S, S D)+U(S, S S)
$$

Thus, when the state is $\omega=S D$, the best 2-plan is not $D D$, since it is dominated by $S S$. So Plan $D$ is not an LFE- $(2,2)$ and Plan $S$ is the sole LFEB-2.

Once again, if decision-makers faced such preferences at a time when their foresight horizon was relatively short (2) they might smoke, then if their foresight horizon later became longer they might again switch to not smoking.

\section{A tale about the smoker's problem:}

In Section 4 we have seen that it is possible to switch the decision of whether to smoke or not smoke simply by changing the horizon of foresight. We propose to interpret this in terms of an (unanticipated) change in foresight over the life-cycle. We suggest that younger people may have less ability to look ahead into the future than older people, and that this offers some insight into why significant numbers of young people take up smoking, only to give it up in middle-life.

Let us think for a moment how relevant our examples are to the problem of why people smoke. How plausible are our payoffs - does our result depend on some very particular arrangement of payoffs which is unlikely to exist in reality?

Think of the payoffs shown in Tables 2 and 3. In all states, the agent instantaneously prefers smoking to non-smoking. Besides, the agent finds smoking relatively more enjoyable (in terms of immediate payoffs) as he has smoked more in the recent past $(U(S, \omega)-U(D, \omega)$ increases as $\omega$ moves from $D D$ to $S S$ through $D S$ and $S D)$.

Consider now the specific payoffs of Table 2. There the least desirable thing to be doing in any period is to stop smoking having smoked in the last two periods $(U(D, S S)=0)$. Anyone who has struggled to stop smoking will attest to the plausibility of this. The highest payoffs come from smoking

${ }^{19} 6+10=16<21=14+7$ 
when smoking has not yet entered a routine stage $(U(S, D D)=U(S, S D)=$ 11). Presumably, since many people go on to be smokers after enduring the unpleasantness of the first cigarette, there is probably a period between just starting and when smoking becomes routine in which smoking is very enjoyable.

Not smoking in any one period having not smoked previously $(U(D, D D)=$ 10 ) is not so attractive as the period just after one has begun smoking (which presumably must be right otherwise who would smoke?), but it is preferable to smoking once smoking has become routine $(U(S, D S)=8 ; U(S, S S)=9)$. As non-smokers themselves, the authors have little difficulty in believing this.

Taking up smoking again after one has only recently stopped $(U(S, D S)=$ $8)$ is more attractive than sticking to not smoking $(U(D, D S)=4)$. Perhaps this is why it is hard to give up?

Similarly, having started smoking, it is pretty unattractive to go back to being a non-smoker $(U(D, S D)=3)$, while smoking is very enjoyable at this point $(U(S, S D)=11)$

In the view of these authors, this set of payoffs seems highly intuitive - indeed compelling. And payoffs of this sort lead to the result that people with a shorter foresight horizon will smoke, while those with a longer foresight horizon (perhaps those who are older and wiser?) will not.

However, we note that the result is not dependent on the ordering of the payoffs so far discussed. In the payoffs shown in Table 4 the ordering is importantly different. In that case, for example, sticking to not smoking having recently given up $(U(D, D S)=10)$ is more attractive than going back to smoking $(U(S, D S)=5)$. Similarly, not continuing with smoking having started $(U(D, S D)=6)$ is higher up the ranking and closer to being a regular smoker $(U(S, S S)=7)$ than in the previous example. An agent with these preferences would appear to find it easier to give up when he wants to. It should hardly be surprising that an agent of this sort might be swayed between smoking and not smoking by the added perspective of a longer foresight horizon.

We should clarify that we do not model any overlap or transition between the young decision-maker's problem with a foresight length of 2 , and the midlife decision-maker's problem with a foresight length of 3. Each problem faced is regarded as an independent infinitely-repeated decision problem. This is clearly an abstraction, but the change in the foresight horizon which our analysis considers helps in understanding how the pattern of smoking may change in the two situations. 


\section{Relation to other approaches}

\subsection{Non-exponential discounting}

What is the relationship between the limited foresight equilibrium approach and the literature on non-exponential discounting as pioneered by Strotz (1956) and revived by Laibson (1997)? The main feature of non-exponential (or hyperbolic) discounting is that the discounting between the payoffs obtained in different periods is not a sole function of the distance between the two periods but also of when these periods actually take place. This literature typically postulates if $\lambda_{t}$ pounds in period $t+k$ are viewed as equivalent to 1 pound in period $t$, the scalar $\lambda_{t}>1$ decreases with $t$.

At first sight the criterion used by an agent with limited foresight has some resemblance with the idea of non-exponential discounting. Indeed the criterion based on limited forecasts as introduced in Definition 1 is as if the agent discounted completely payoffs obtained beyond his horizon of foresight. Yet, an alternative and equivalent (and in our opinion preferable) view is that the agent believes (possibly erroneously) that the pattern of payoffs obtained within his horizon of foresight will repeat infinitely also beyond his horizon of foresight and that he uses his normal preference with exponential discounting to compare the effect of the various possible actions. Clearly, with this alternative interpretation, the criterion used by the agent does not exhibit the kind of non-exponential discounting present in the previously mentioned literature. But (unlike in the hyperbolic discounting literature) the agent's expectations beyond the horizon of foresight may be erroneous, as there is no guarantee that the streams of payoffs obtained within the horizon of foresight will repeat infinitely beyond the horizon of foresight.

We might have considered alternative decision-making criteria based on the limited horizon forecast. For example, we might have parameterized agents also by their degree of optimism regarding their assessment of what they do not predict. Or in the line with Jehiel (2001) we might have considered the possibility that agents' criteria are also affected by the realization of their state of mind regarding how they assess what is to come beyond their horizon of foresight. We have chosen the criterion based on the simple extrapolation idea because it allows to express our point simply, but the main insight of our paper (regarding the effect of a change in the horizon of foresight) would also hold for the alternative criteria just mentioned (for which there would be no interpretation of the criterion based on non-exponential 
discounting).

As a final comment on the difference between limited foresight and hyperbolic discounting, observe that in our setup the underlying preferences are completely standard, and the change in horizons of foresight concerns solely the cognitive abilities of the agents. Hence the notion of the well-being of the agent is unambiguously defined in our setting, unlike in the hyperbolic discounting case in which welfare analysis always depends on the criterion chosen by the planner (the choice of which time is the right one). This means that there is no difficulty here of speaking of the effect of a change in the horizon of foresight on the well-being of the agent.

\subsection{Addiction theories}

In the Stigler and Becker (1977) tradition, there have been a number of approaches based on "rational addiction", whereby people employ "appreciation capital" which is affected by consuming. Becker et al. (1994) find some empirical evidence for such rational addiction in cigarette consumption where they establish that sales of cigarettes in the current period decreases if future prices increase, which they interpret as complementarities between current and future cigarette consumption (see also Gruber and Koszegi (2001) for further test of this assumption).

In our model, agents are not fully rational; yet their preferences depend on past consumption (through the dependence of $U(a, \omega)$ on the state $\omega$ ). When $U(C, \omega)-U(D, \omega)$ increases as the consumer has smoked more in the past (which is reflected in the state $\omega$ ), we are inclined to say that our consumer's preferences exhibit addiction (see the discussion surrounding the examples in Section 3). By introducing bounded rationality, we are able to explain a change of consumption path as a result of a change of horizon of foresight, which we think complements in a new way the literature on rational addiction.

In a recent paper, Gul and Pesendorfer (2007) proposes a new theory of harmful addiction in which the mere possibility of cigarette consumption hurts the agent whether or not he smokes. As argued by the authors, in such a case taxing cigarettes is not as effective as banning cigarettes as in the former case the agent has still the option of consuming cigarettes (the fact that it is made less attractive can only hurt the agent in Gul-Pesendorfer's model). To some extent, Gul-Pesendorfer's theory can be viewed as revisiting the classic theory of addiction when preferences are not defined over consumptions but 
over consumption opportunities. This is complementary to our approach, which can be viewed as revisiting the classic theory of addiction by adding some element of cognitive limitations.

\subsection{Other cognitive biases}

The literature on behavioural economics has emphasized a number of cognitive biases other than limited foresight. These in particular include the idea of projection bias in which agents exaggerate the degree to which their future tastes will resemble their current tastes. Loewenstein et al (2003) have pursued this idea to explain consumption decisions that are generally attributed to time inconsistency. In particular, they apply their model to show why agents who are subject to projection bias may smoke too much early in life minimizing the danger of addiction. To illustrate this idea in the context of the model of Section 2, an agent subject to projection bias (as modelled by Loewenstein et al.) would expect future states $\omega_{t^{\prime}}$ to coincide with the current state $\omega_{t}$ with some positive probability and he would have the correct expectation about the period $t^{\prime}$ state otherwise. A limiting case of this model is when the agent thinks the state will stay the same as it is now in which case the agent chooses the optimal myopic decision (or the LFE-1 plan in the language of this paper). Another limiting case is when the agent has perfect foresight, which corresponds to the rational solution. Intermediate cases like the LFE- $n$ concept correspond to cases that are in between the myopic case and the fully rational case. However, the cognitive limitations associated with limited foresight and projection bias are very different. To illustrate one, limited foresight assumes that agents' forecasts are correct even though limited, which allows us to justify the limited foresight equilibrium concept by a learning story. It is unclear how to interpret Loewenstein et al.'s model of projection bias given that the agent's theory is hard to interpret from a limited learning perspective. Besides, our focus is on how improving cognitive abilities (through longer horizons of foresight) may change the pattern of consumption of goods like cigarettes, which does not seem to have its counterpart in Loewenstein et al.

Finally, we should mention the work of O'Donoghue and Rabin (1999) which combines both the idea of hyperbolic discounting as a true description of the underlying preferences and the idea of partial sophistications to describe the cognitive abilities of the agents. In their approach, a sophisticated agent is one who is aware of his limited control capabilities and of the nature 
of his preferences. A naive agent is one who behaves as if he could stick to his plan afterwards, which in reality he cannot, and partial sophistication is modelled as a convex combination between the two extreme modes of behavior outlined above. One should mention that the O'Donoghue and Rabin approach to partial sophistication and the limited foresight approach are very different since again it is not clear how to interpret the partial sophistication in O'Donoghue and Rabin approach from a limited learning perspective (so the question of how a partially sophisticated agent would behave as in O'Donoghue and Rabin approach seems insufficiently justified to us).

One other advantage of our approach over that employed by O'Donoghue and Rabin is that the notion of the well-being of the agent is unambiguously defined in our setting, unlike in the hyperbolic discounting case in which welfare analysis always depends on the criterion chosen by the planner (the choice of which time is the right one). Relatedly, the two approaches lead to different policy recommendations. In our case a fully rational agent is always better off than a partially rational agent, which need not be so in O'Donoghue and Rabin's approach. It follows that increasing the cognitive ability of the agent by for example making agents more aware of the usual consumption patterns (social learning) seems always desirable in our framework while it may be detrimental in O'Donoghue and Rabin's approach.

\section{Conclusion}

In this paper we have offered one interpretation of why people start smoking only to give it up later: as people grow older they gain a longer-term perspective which changes the balance of advantage away from smoking and towards not smoking. We contend that the preferences required to obtain this result are interpreted plausibly in terms of scenarios in which people start smoking only to give it up later.

The limited foresight perspective adopted in this paper is not merely a reinterpretation of hyperbolic discounting models. We believe it suggests policy implications that are very different from those that derive from the hyperbolic discounting literature. One clear way to improve the well-being of agents in the present framework is to inform them better about the effects of their choices and the likely consumption patterns that follow from their current consumption. That is, increasing agents' horizon of foresight is always beneficial. With the hyperbolic discounting view, policy interventions are 
viewed in a very different way: cigarettes should be taxed so as to better align the incentive of the current self with the long term well-being of the agent. But, a uniform tax applied to agents with different taste parameters and also different horizons of foresight may be detrimental in our context with limited foresight and exponential discounting (in particular, those who have perfect foresight can only suffer from a tax as in Gul and Pesendorfer's theory of harmful addiction). We believe more experimental/empirical work should be devoted to the issue of disentangling the taste preferences of the agents from their cognitive limitations so as to better design policy interventions.

\section{References}

[1] Ainslee, G. [1975], "Specious reward: A behavioral theory of impulsiveness and impulse control" , Psychological Bulletin 82(4), 463-496

[2] Becker, G.S. and K.M. Murphy [1988], "A theory of rational addiction", Journal of Political Economy, 675-700

[3] Becker, G.S., Grossman, M. and K.M. Murphy [1994], "An empirical analysis of cigarette addiction", American Economic Review, 84, 396418

[4] Bernheim, D., Ray, D. and Yeltekin, S. [1999], "Self-control, saving and the low asset trap" , mimeo Stanford

[5] Gruber, J. and B. Koszegi [2001], "Is addiction rational? Theory and evidence", Quarterly Journal of Economics, 116, 1261-1303

[6] Gul, F and Pesendorfer, W. [2007], "Harmful Addiction," Review of Economic Studies, 74, 147-172.

[7] Harris, C. and Laibson D. [2001], "Dynamic choices of hyperbolic consumers," Econometrica 69, 935-958

[8] Jehiel, P. [1995], "Limited horizon forecast in repeated alternate games," Journal of Economic Theory 67, 497-519

[9] Jehiel, P. [1998a], "Learning to play limited foresight equilibria," Games and Economic Behaviour 22, 274-298 
[10] Jehiel, P. [2001], 'Limited foresight may force cooperation,' Review of Economic Studies, April 2001

[11] Laibson, David [1997], "Golden Eggs and Hyperbolic Discounting", Quarterly Journal of Economics, 443-477

[12] Lilico, A. [2002], "Limited foresight", Ph.D. Thesis, University College, London

[13] Loewenstien, G., O'Donoghue, T. and M. Rabin [2003], "Projection Bias in Predicting Future Utility", Quarterly Journal of Economics, 118, $1209-1248$

[14] O'Donoghue, T. and M. Rabin [1999], "Doing it now or later," American Economic Review 89(1), 103-124

[15] O'Donoghue, T. and M. Rabin [2001]. "Choice and Procrastination," Quarterly Journal of Economics 116, 121-160

[16] Rubinstein, A. [1998], "Modeling bounded rationality," MIT Press.

[17] Stigler, G.J. \& Becker, G.S. [1977], "De gustibus non est disputandum," American Economic Review 67(2), 76-90

[18] Strotz, R.H. [1956], "Myopia and inconsistency in dynamic utility maximization," Review of Economic Studies 23, 165-180 


\section{Appendix}

Proof of Lemma1:

Proof. Assume, without loss of generality, that $a=D$ (we could simply reverse symbols if $a=S)$.

If Plan $D$ is an LFE- $(m+1)$ then the following $2^{m}$ inequalities must hold $\left(2^{m}\right.$ because there is one inequality for each possible state at time $t$, and there are $2^{m}$ different possible states):

1) $(m+1) U(D, D \ldots D) \geq U(S, D \ldots D)+U(D, S D \ldots D)+\ldots+U(D, D \ldots D S)$

2) $U(D, S D \ldots D)+\ldots+U(D, D \ldots D) \geq U(S, S D \ldots D)+\ldots+U(D, D \ldots D S)$

.

$\left.2^{m}\right) U(D, S \ldots S)+\ldots+U(D, D \ldots D) \geq U(S, S \ldots S)+\ldots+U(D, D \ldots D S)$

These inequalities state that, for every possible state at $t$, it is better to play $D$ this period than to play $S$, on the assumption that $D$ will be played thereafter.

If Plan $D$ is an LFE- $n$ there will again be $2^{m}$ inequalities (only $2^{m}$ because there are still only $2^{m}$ possible states, even though there is now greater foresight). Now the sum of the payoffs from playing $D$ this period (the lefthand side) for any state at $t$ would differ from the inequalities above by the presence of an additional $n-m-1$ added terms of $U(D, D \ldots D)$. But the sum of payoffs from playing $S$ this period (the right hand side) would, likewise, differ only by the presence of the same additional $n-m-1$ added terms of $U(D, D \ldots D)$.

Thus, for example, inequality (2) would be modified to read

$\left.2^{\prime}\right) U(D, S D \ldots D)+\ldots+U(D, D \ldots D)+(n-m-1) U(D, D \ldots D) \geq$

$U(S, S D \ldots D)+\ldots+U(D, D \ldots D S)+(n-m-1) U(D, D \ldots D)$

but this simplifies back to equation (2). Hence the required inequalities are equivalent, and whenever they are satisfied Plan $D$ will be both an LFE$(m+1)$ and an LFE- $n$, and whenever they are not all satisfied, Plan D will be neither an LFE- $(m+1)$ nor a LFE- $n$.

Proof of Lemma 2:

Proof. If Plan $D$ is optimal with perfect foresight, then the infinite stream of payoffs received from playing $D$ every period must be greater than that from playing $S$ this period then playing $D$ thereafter, for any state. But 
after period $t+m$, if $D$ is being played from period $t+1$, all subsequent payoffs will be $U(D, D \ldots D)$, regardless of whether $D$ or $S$ is played in period $t$ since there are only $m$ elements in the state variable. That means that when Plan $D$ is optimal with perfect foresight, the $2^{m}$ inequalities of the proof of Lemma 1 must hold, which is a sufficient condition for Plan $D$ to be an $\operatorname{LFE}-(m+1)$.

Proof of Proposition 2:

Proof. (Sketch) Note that since (by assumption) Plan $S$ is not optimal with perfect foresight, it cannot be true that a set of $2^{m}$ inequalities equivalent to those in the proof of Lemma 1 hold. Hence Plan $S$ is not an LFE- $(m+1)$. By Lemma 1 this implies that Plan $S$ is not an LFE- $n$ for any $n>m+1$ either. This should help to verify Proposition 2. 


\section{CESifo Working Paper Series}

for full list see www.cesifo-group.org/wp

(address: Poschingerstr. 5, 81679 Munich, Germany, office@cesifo.de)

2541 Philipp C. Bauer and Regina T. Riphahn, Age at School Entry and Intergenerational Educational Mobility, February 2009

2542 Thomas Eichner and Rüdiger Pethig, Carbon Leakage, the Green Paradox and Perfect Future Markets, February 2009

2543 M. Hashem Pesaran, Andreas Pick and Allan Timmermann, Variable Selection and Inference for Multi-period Forecasting Problems, February 2009

2544 Mathias Hoffmann and Iryna Shcherbakova, Consumption Risk Sharing over the Business Cycle: the Role of Small Firms' Access to Credit Markets, February 2009

2545 John Beirne, Guglielmo Maria Caporale, Marianne Schulze-Ghattas and Nicola Spagnolo, Volatility Spillovers and Contagion from Mature to Emerging Stock Markets, February 2009

2546 Ali Bayar and Bram Smeets, Economic and Political Determinants of Budget Deficits in the European Union: A Dynamic Random Coefficient Approach, February 2009

2547 Jan K. Brueckner and Anming Zhang, Airline Emission Charges: Effects on Airfares, Service Quality, and Aircraft Design, February 2009

2548 Dolores Messer and Stefan C. Wolter, Money Matters - Evidence from a Large-Scale Randomized Field Experiment with Vouchers for Adult Training, February 2009

2549 Johannes Rincke and Christian Traxler, Deterrence through Word of Mouth, February 2009

2550 Gabriella Legrenzi, Asymmetric and Non-Linear Adjustments in Local Fiscal Policy, February 2009

2551 Bruno S. Frey, David A. Savage and Benno Torgler, Surviving the Titanic Disaster: Economic, Natural and Social Determinants, February 2009

2552 Per Engström, Patrik Hesselius and Bertil Holmlund, Vacancy Referrals, Job Search, and the Duration of Unemployment: A Randomized Experiment, February 2009

2553 Giorgio Bellettini, Carlotta Berti Ceroni and Giovanni Prarolo, Political Persistence, Connections and Economic Growth, February 2009

2554 Steinar Holden and Fredrik Wulfsberg, Wage Rigidity, Institutions, and Inflation, February 2009

2555 Alexander Haupt and Tim Krieger, The Role of Mobility in Tax and Subsidy Competition, February 2009 
2556 Harald Badinger and Peter Egger, Estimation of Higher-Order Spatial Autoregressive Panel Data Error Component Models, February 2009

2557 Christian Keuschnigg, Corporate Taxation and the Welfare State, February 2009

2558 Marcel Gérard, Hubert Jayet and Sonia Paty, Tax Interactions among Belgian Municipalities: Does Language Matter?, February 2009

2559 António Afonso and Christophe Rault, Budgetary and External Imbalances Relationship: A Panel Data Diagnostic, February 2009

2560 Stefan Krasa and Mattias Polborn, Political Competition between Differentiated Candidates, February 2009

2561 Carsten Hefeker, Taxation, Corruption and the Exchange Rate Regime, February 2009

2562 Jiahua Che and Gerald Willmann, The Economics of a Multilateral Investment Agreement, February 2009

2563 Scott Alan Carson, Demographic, Residential, and Socioeconomic Effects on the Distribution of $19^{\text {th }}$ Century US White Statures, February 2009

2564 Philipp Harms, Oliver Lorz and Dieter Urban, Offshoring along the Production Chain, February 2009

2565 Patricia Apps, Ngo Van Long and Ray Rees, Optimal Piecewise Linear Income Taxation, February 2009

2566 John Whalley and Shunming Zhang, On the Arbitrariness of Consumption, February 2009

2567 Marie-Louise Leroux, Endogenous Differential Mortality, Non-Contractible Effort and Non Linear Taxation, March 2009

2568 Joanna Bęza-Bojanowska and Ronald MacDonald, The Behavioural Zloty/Euro Equilibrium Exchange Rate, March 2009

2569 Bart Cockx and Matteo Picchio, Are Short-Lived Jobs Stepping Stones to Long-Lasting Jobs?, March 2009

2570 David Card, Jochen Kluve and Andrea Weber, Active Labor Market Policy Evaluations: A Meta-analysis, March 2009

2571 Frederick van der Ploeg and Anthony J. Venables, Harnessing Windfall Revenues: Optimal Policies for Resource-Rich Developing Economies, March 2009

2572 Ondřej Schneider, Reforming Pensions in Europe: Economic Fundamentals and Political Factors, March 2009 
2573 Jo Thori Lind, Karl Ove Moene and Fredrik Willumsen, Opium for the Masses? Conflict-Induced Narcotics Production in Afghanistan, March 2009

2574 Silvia Marchesi, Laura Sabani and Axel Dreher, Agency and Communication in IMF Conditional Lending: Theory and Empirical Evidence, March 2009

2575 Carlo Altavilla and Matteo Ciccarelli, The Effects of Monetary Policy on Unemployment Dynamics under Model Uncertainty - Evidence from the US and the Euro Area, March 2009

2576 Falko Fecht, Kjell G. Nyborg and Jörg Rocholl, The Price of Liquidity: Bank Characteristics and Market Conditions, March 2009

2577 Giorgio Bellettini and Filippo Taddei, Real Estate Prices and the Importance of Bequest Taxation, March 2009

2578 Annette Bergemann and Regina T. Riphahn, Female Labor Supply and Parental Leave Benefits - The Causal Effect of Paying Higher Transfers for a Shorter Period of Time, March 2009

2579 Thomas Eichner and Rüdiger Pethig, EU-Type Carbon Emissions Trade and the Distributional Impact of Overlapping Emissions Taxes, March 2009

2580 Antonios Antypas, Guglielmo Maria Caporale, Nikolaos Kourogenis and Nikitas Pittis, Selectivity, Market Timing and the Morningstar Star-Rating System, March 2009

2581 António Afonso and Christophe Rault, Bootstrap Panel Granger-Causality between Government Budget and External Deficits for the EU, March 2009

2582 Bernd Süssmuth, Malte Heyne and Wolfgang Maennig, Induced Civic Pride and Integration, March 2009

2583 Martin Peitz and Markus Reisinger, Indirect Taxation in Vertical Oligopoly, March 2009

2584 Petra M. Geraats, Trends in Monetary Policy Transparency, March 2009

2585 Johannes Abeler, Armin Falk, Lorenz Götte and David Huffman, Reference Points and Effort Provision, March 2009

2586 Wolfram F. Richter, Taxing Education in Ramsey’s Tradition, March 2009

2587 Yin-Wong Cheung, Menzie D. Chinn and Eiji Fujii, China's Current Account and Exchange Rate, March 2009

2588 Alexander Haupt and Silke Uebelmesser, Voting on Labour-Market Integration and Education Policy when Citizens Differ in Mobility and Ability, March 2009

2589 Hans Jarle Kind, Marko Koethenbuerger and Guttorm Schjelderup, Should UtilityReducing Media Advertising be Taxed?, March 2009 
2590 Alessandro Cigno, How to Avoid a Pension Crisis: A Question of Intelligent System Design, March 2009

2591 Helmut Lütkepohl and Fang $\mathrm{Xu}$, The Role of the Log Transformation in Forecasting Economic Variables, March 2009

2592 Rainald Borck, Hyun-Ju Koh and Michael Pflüger, Inefficient Lock-in and Subsidy Competition, March 2009

2593 Paolo M. Panteghini, On the Equivalence between Labor and Consumption Taxation, March 2009

2594 Bruno S. Frey, Economists in the PITS?, March 2009

2595 Natalie Chen and Dennis Novy, International Trade Integration: A Disaggregated Approach, March 2009

2596 Frédérique Bec and Christian Gollier, Term Structure and Cyclicity of Value-at-Risk: Consequences for the Solvency Capital Requirement, March 2009

2597 Carsten Eckel, International Trade and Retailing, March 2009

2598 Gianni De Nicolò and Iryna Ivaschenko, Global Liquidity, Risk Premiums and Growth Opportunities, March 2009

2599 Jay Pil Choi and Heiko Gerlach, International Antitrust Enforcement and Multi-Market Contact, March 2009

2600 Massimo Bordignon and Guido Tabellini, Moderating Political Extremism: Single Round vs Runoff Elections under Plurality Rule, April 2009

2601 Ana B. Ania and Andreas Wagener, The Open Method of Coordination (OMC) as an Evolutionary Learning Process, April 2009

2602 Simon Gächter, Daniele Nosenzo, Elke Renner and Martin Sefton, Sequential versus Simultaneous Contributions to Public Goods: Experimental Evidence, April 2009

2603 Philippe Jehiel and Andrew Lilico, Smoking Today and Stopping Tomorrow: A Limited Foresight Perspective, April 2009 